\title{
Hudelist, Andreas \& Krammer, Stefan (Hrsg.) (2017): ide, 41/3. Innsbruck: StudienVerlag
}

\section{Dragan Miladinović}

Die Ausgabe 3/2017 der österreichischen, deutschdidaktischen Zeitschrift ide nimmt die Kultur des Performativen in den Blick und setzt damit eine Themenreihe zu anderen, in der Vergangenheit bereits abgehandelten Kulturen des Deutschunterrichts (u.a. des Sehens, des Hörens) fort. Im Performativen sehen die Herausgeber Andreas Hudelist und Stefan Krammer im Sinne des performative turn „weniger ein neues Phänomen als eine andere Art und Weise, bekannte Phänomene zu betrachten, zu begreifen, zu reflektieren“ (5) und genau da setze diese Ausgabe der vierteljährig erscheinenden Zeitschrift an: die Deutschdidaktik und ihre „unterschiedlichen Kulturen des Aus- und Aufführens" (5) aus performativer Sicht zu perspektivieren. Dabei geht es beispielsweise darum, wie Sprache beim Schreiben und Sprechen ausgeführt und theatrale und soziale Handlungen schulisch aufgeführt bzw. wie „Körperlichkeit und Materialität bei der Vermittlung von Sprache und Literatur“ (6) inszeniert werden. Angemerkt sei hier, dass performativ in ide nicht wie von Schewe (u.a. 2013) vorgeschlagen als Schirmbegriff für jegliches Lehren, Lernen und Forschen dient, das sich Inspirationen aus den performativen Künsten einholt. Performativ bedeutet hier, dass „Ausführungs-, Inszenierungsund Aufführungspraktiken sozialen Handelns, deren wirklichkeitskonstitutive Prozesse wie auch das Verhältnis von Sprache, Körper, Sozialität und Macht in den Blick genommen [werden]" (6). Gleichzeitig ist aber für beide die Theaterwissenschaft eine wichtige Bezugsdisziplin, insofern finden sich auch trotz der unterschiedlichen Verwendung Überschneidungspunkte.

Das Heft ist grob dreigeteilt in einen (begriffs-)theoretischen, einen didaktischen sowie einen praxisbezogenen Abschnitt. Abgerundet wird die Ausgabe mit einem bibliographischen Überblick zu Forschungsbeiträgen im Bereich des Performativen. Den Abschluss mit dem für die Zeitschrift typischen Magazinteil bilden ein Unterrichtsvorschlag zum Thema Metaphern (111-115), ein Kommentar zu Bildungsstandards, Schulreformen und Bildungsforschung (116-118), eine Buch- und Veranstaltungsempfehlung sowie Buchrezensionen (119-128).

Im ersten Teil wird die wichtige, theoretische Grundlage gelegt, die besonders für die vielfältig und intradisziplinär verwendeten Begriffe des Performativen wichtig sind, denn gerade diese Vielfalt würde die Verortung der Beiträge ohne diese Einführung erschweren. Den Anfang leistet die historische und durch den Autor als „unvollständig“ markierte Genese der drei Begriffe Performanz, 
Performativität und Performance von Andreas Hudelist. Die Erkundung führt an die „Theorien des Performativen“ "heran, u.a. von Austin, über Searle, Butler und Erika Fischer-Lichte zu McKenzie, und regt zur weiteren Auseinandersetzung mit den Begriffen ein. Er lässt sich gut mit anderen begrifflichen Erkundungen lesen (vgl.bspw. Fleming 2016) und der im Heft enthaltene bibliographische Überblick bietet sich dafür als erste Anlaufstelle an. Jörg Zirfas‘ Beitrag beschäftigt sich mit der Bedeutung von Performativität in der Pädagogik, indem er dies anhand von sieben Punkten verdeutlicht. Zugrunde liegt die Annahme, dass Handeln „als Nachahmung, Teilnahme und Gestaltung kultureller Praktiken“ (20) verstanden wird und „[d]azu gehören: Künstlerisches und soziales Handeln als performance, Sprechen als performatives Handeln und Performativität als ein abgeleiteter, diese Zusammenhänge übergreifend thematisierender Begriff." (20, Herv. i. O.) Seine Schlussfolgerung für die Lehrausbildung lautet, dass Lehrpersonen „sowohl eine objektive Inhalts- und Methodenkompetenz als auch eine subjektive Handlungskompetenz" (27) benötigten, weswegen ein Schwerpunkt auf der Ausbildung der sinnlichen und körperlichen sowie mimetischen Dimension liegen sollte.

Bezugnehmend auf die vorgestellten Theorien entwickelt Stefan Krammer dann „Perspektiven einer performativen Deutschdidaktik“ (30), indem er sowohl für sprachliches, literarisches als auch identitätsbezogenes Arbeiten performative Ansätze skizziert. Dabei gehe es einer performativen Deutschdidaktik neben der sprachlichen und literarästhetischen Aus- und Aufführung auch um die soziale Handlung, d.h. „die Ich-Entwicklung, das Einnehmen verschiedener Rollen und die Konstruktion von Identität" (35).

Konkretere Beispiele, wie sich eine performative Deutschdidaktik realisieren lassen könnte, finden sich in den beiden folgenden Abschnitten der Zeitschrift. Der zweite Teil offenbart performative, didaktische Überlegungen: Eine auf die Körperlichkeit und aufs ästhetische Lernen bezogene Wahrnehmungsschulung liefert Gabriela Paule (40-48), Jürgen Struger betrachtet das Schreiben als performativen Akt (49-58) und hebt Schreiben als „schreibendes Handeln“ (57) hervor. Matthias Leichtfried diskutiert seine theoretischen Überlegungen rund um das literarische Gespräch als Aufführung, bei denen sich Hot-Seating (vgl. bspw. Even 2018) als praktische Umsetzungsform gut eignen müssten. Carolin Führer geht auf den (multimodalen) Mehrwert ein, den szenisches Lesen von Comics haben kann.

Der letzte Teil liefert Einblicke in die (außer-)schulische Praxis. Julia Malle (75-83) zeichnet ein Unterrichtsmodell nach, in dem mit theaterdidaktischen Ansätzen an der Entwicklung von Gendersensibilität gearbeitet wird. Die professionelle Erzählerin Christine Lander (84-94) berichtet u.a. vom LangzeitErzählprojekt ErzählZeit und von den Vorteilen des freien Erzählens gegenüber dem Vorlesen, da die gehörte Sprache zusätzlich mit Gesten unterstützt werde. Im letzten Beitrag dieses Abschnitts zeigen Markus Köhle und Mieze Medusa (95-101), welche performativen Elemente bei Poetry Slams zum Tragen kommen und liefern Antworten zum Thema Poetry Slam sowie Tipps für einen „gelungen Auftritt“ (100). 
Insgesamt bietet diese Ausgabe viele Inspirationsquellen für performatives Arbeiten im Deutschunterricht. Etwas überraschend ist vielleicht, gerade für Scenario-Leser*innen, dass trotz der Vielzahl der angeschnitten Fachdiskurse, inklusive der Theaterpädagogik, jener zur Dramapädagogik vernachlässigt wird und sich weder in den Beiträgen, noch in dem bibliographischen Überblick finden lässt - und das obwohl sich viele Überschneidungen finden ließen. Insofern wird auch das Scenario-Projekt als Quelle für performatives Arbeiten leider nicht erwähnt. Dies mag zwar an der zumeist fremdsprachendidaktischen Verortung der Dramapädagogik liegen, dennoch ließen sich sicherlich auch für die Deutschdidaktik lohnende Perspektiven entdecken.

\section{Bibliografie}

Even, Susanne (2018): Nothing moves if you don't let go. Performativity in teacher training. In: Mentz, Olivier Mentz \& Fleiner, Micha (Hrsg.): The Arts in Language Teaching. International Perspectives: Performative - Aesthetic Transversal. Berlin u.a.: Lit-Verlag, 90-106

Fleming, Mike (2016): Überlegungen zum Konzept performativen Lehrens und Lernens. In: Even, Susanne \& Schewe, Manfred (Hrsg.): Performatives Lehren, Lernen, Forschen. Performative Teaching, Learning, Research. Berlin/Stuttgart/Milow: Schibri, 27-46

Schewe, Manfred (2013): Taking Stock and Looking Ahead: Drama Pedagogy as a Gateway to a Performative Teaching and Learning Culture. In Scenario VII/1, 5-23. http://research.ucc.ie/scenario/2013/01/Schewe/02/en [letzter Zugriff: 10.12.2018] 